\title{
TENDENCIAS EN EL TRATAMIENTO DE AGUAS RESIDUALES DOMÉSTICAS
}

Patricia Torres*

* Ph.D. en Ingeniería Civil, Universidad de Sao Paulo, Brasil.

Profesora de la Escuela de Ingeniería de Recursos Naturales y del Ambiente Facultad de Ingeniería - Universidad del valle.

\section{RESUMEN}

El aumento de la conciencia de que el tratamiento del agua residual es de importancia vital para evitar o reducir la contaminación ambiental, ha resultado en la necesidad de desarrollar procesos que combinen una alta eficiencia de tratamiento con bajos costos de construcción y de operación y mantenimiento. Este trabajo pretende mostrar las nuevas tendencias en el tratamiento de aguas residuales domésticas, buscando la aplicación de tecnologías apropiadas a nuestro medio desde el punto de vista tanto técnico como económico y de eficiencia de remoción para obtener un efluente tratado de buena calidad. Se presentan 
algunas de las experiencias de mayor aplicación tanto en el país como en regiones con características similares desde el punto de vista socio-económico como climático. Se considera que la aplicación de la tecnología anaerobia combinada con diferentes opciones aerobias como unidad complementaria de los sistemas de tratamiento, ofrecen una alternativa interesante para nuestro medio por obtenerse efluentes de buena calidad con menores costos tanto de inversión como de operación y mantenimiento comparados con la aplicación de la tecnología aerobia convencional.

Palabras clave: agua residual doméstica; tratamiento aerobio; postratamiento; sistemas combinados

\section{ABSTRACT}

The increasing consciousness that wastewater treatment is of vital importance to avoid or to reduce the environmental contamination, resulted in the necessity to develop processes that combine a high treatment efficiency with low construction and operation and maintenance costs. This work intends to show the new tendencies on domestic sewage treatment applying appropriate technologies of the technical and economical viewpoint and removal efficiency to obtain a good quality in the treated effluent. There are shown any experiences of most application in our country and on regions with similar social-economical and climatic characteristics. It is considered that the application of the anaerobic technology combined with different aerobic options as complementary unity of the treatment systems, offer an interesting alternative for our country because it is possible to obtain effluents with a good quality with less cost on investment and operation and maintenance if compared with the application of conventional aerobic technology.

Keywords: domestic sewage; anaerobic treatment; post-treatment; combined systems.

\section{INTRODUCCIÓN}

Existe gran variedad de sistemas que pueden ser aplicados para el tratamiento de aguas residuales; sin embargo, mientras en los países desarrollados el número de alternativas es limitado debido a que los estándares de calidad de los efluentes usualmente aplicados son más rígidos, en los países en desarrollo en general las posibilidades pueden ser mucho mayores debido a la diversidad de criterios de calidad de efluentes, al alto contraste entre áreas urbanas, periféricas y rurales y a factores como costos y requerimientos operacionales, decisivos en la selección de alternativas en estos países.

Grandes Plantas de Tratamiento de Aguas Residuales PTAR, del tipo lodos activados convencionales frecuentemente no son adecuadas en algunos lugares debido a la limitada disponibilidad de área, recursos humanos, etc. En algunos casos, la tecnología de tratamiento anaerobio en sus diversas formas, se presenta no como la única ni como la mejor, sino como la más viable por tener bajo costo, ser sostenible y eficiente en la remoción de compuestos orgánicos biodegradables, aunque compuestos mineralizados como nitrógeno amoniacal, fosfatos y sulfuros y microorganismos patógenos, permanecen en el efluente y deben ser removidos en una etapa adicional de postratamiento.

Dentro de los sistemas anaerobios, los reactores UASB (Up flow Anaerobic Sludge Blanket: Reactor Anaerobio de Manto de Lodos y Flujo Ascendente) y sus variaciones, han sido los de mayor aplicabilidad en el tratamiento de agua residual doméstica, con eficiencia de remoción de materia orgánica razonablemente buena y a un costo relativamente bajo. En América Latina y otros países de clima tropical y templado, donde ocurre implementación creciente de la tecnología anaerobia, la adopción del reactor anaerobio seguido de un reactor aerobio para pos- 
tratamiento, se constituye en una alternativa atrayente en lo que se refiere a los aspectos económicos y ambientales.

Algunos reactores ya poseen tratamiento complementario con sistemas como disposición en el suelo, lagunas de estabilización, filtros biológicos, lodos activados de flujo continuo o intermitente con estabilización del lodo aerobio en el reactor UASB, etc. Estos sistemas complementarios, permiten remoción adicional de materia orgánica y algunos también favorecen la nitrificación del efluente final. En este artículo se presentan algunas de las combinaciones más aplicadas.

\section{LA TECNOLOGÍA DE REACTORES A N A E R O B IOS P A R A E L TRATAMIENTO DE AGUA RESIDUAL DOMÉSTICA}

La mayoría (89.7\%) de los sistemas anaerobios existentes, son utilizados para el tratamiento de efluentes industriales, mientras que apenas $7.3 \%$ tratan agua residual doméstica y $3.0 \%$ tratan residuos sólidos orgánicos (GTZ, 1997).

La temperatura es un factor ambiental de gran importancia para el buen funcionamiento del sistema. Las regiones en las que ha ocurrido mayor aplicabilidad de esta tecnología son las de clima tropical y subtropical, donde la temperatura es siempre mayor que $20^{\circ} \mathrm{C}$. En el rango de 12 a $20^{\circ} \mathrm{C}$ existen diversas experiencias que demuestran que el proceso también es viable pero deben determinarse condiciones óptimas de diseño y mayor control en el proceso.

En América Latina, los países con mayor grado de aplicación en escala real son Brasil, Colombia, México y Ecuador. Otros países que han implementado grandes plantas de tratamiento son China e India. Egipto también viene utilizando la tecnología.
Otro aspecto interesante de la tecnología anaerobia es que puede ser usada también en escala pequeña, factor de importancia en los países en desarrollo donde es necesario descentralizar los sistemas para reducir costos. El Estado de Paraná en Brasil, es un buen ejemplo de esta situación, donde se tienen instaladas más de 1000 unidades de pequeño porte (PROSAB, 1998).

Las principales características de los reactores tipo UASB que tratan agua residual doméstica son:

Rango de temperatura media de operación:

$$
12-30^{\circ} \mathrm{C}
$$

Rango de temperatura óptima de operación:

$$
20-30^{\circ} \mathrm{C}
$$

Tiempo de retención hidráulico:

$$
4-12 h
$$

Carga orgánica volumétrica:

$$
<6 \mathrm{~kg} \mathrm{DQO} / \mathrm{m}^{3} \mathrm{~d}
$$

Eficiencia remoción $\mathrm{DQO}_{\text {total }}$ y $\mathrm{DBO}_{\text {total }}$ :

$$
60-80 \%
$$

Eficiencia remoción $\mathrm{DQ} \mathrm{O}_{\text {filtrada }}, \mathrm{DBO}_{\text {filtrada }}$ :

$$
75-85 \%
$$

Eficiencia Remoción Sólidos Suspendidos:

$$
70-80 \%
$$

Eficiencia Remoción MTK:

$$
10-25 \%
$$

Eficiencia remoción $\mathrm{P}$ :

$$
10-20 \%
$$

En términos generales, las remociones de la materia orgánica carbonácea (DBO, DQO) y de los sólidos suspendidos ocurren con una buena eficiencia en el reactor anaerobio; sin embargo, la remoción de nutrientes y de microorganismos patógenos es mínima, lo que amerita la necesidad de implementar sistemas complementarios de tratamiento.

En algunos casos, dependiendo de la disposición final del efluente y de la legislación local sobre la calidad mínima del mismo, los niveles de 
tratamiento que se alcanzan con la etapa anaerobia, son suficientes. Cuando es necesario utilizar tratamiento aerobio como complemento al anaerobio, los costos de los procesos aerobios son minimizados, resultando el sistema combinado anaerobio-aerobio una alternativa mucho más económica que la alternativa del sistema aerobio único.

Cuando se pretende remover sólo materia orgánica carbonácea, apenas los sistemas compuestos de tanque séptico seguido de filtro anaerobio o de reactor UASB seguido de filtro anaerobio, que sólo son viables para poblaciones pequeñas, generalmente inferiores a 1000 habitantes, no requieren del uso de sistemas aerobios para responder al límite de concentración exigido (PROSAB, 1998).

\section{POSTRATAMIENTO DE EFLUENTES DE REACTORES ANAEROBIOS}

Los objetivos principales del postratamiento son (VIÑAS, 1994):

- Aumentar la confiabilidad de estos sistemas.

- Mejorar la eficiencia del proceso global de eliminación del carbono.

> Mejorar la separación de los sólidos.

> Introducir procesos de eliminación de nutrientes.

> Disponer adecuadamente los residuos sólidos generados en el proceso global.

El tratamiento anaerobio puede ser complementado con métodos físicos o químicos, biológicos o una combinación de estos, para obtener remoción adicional de DBO, sólidos, nutrientes o microorganismos patógenos. Las principales alternativas para el tratamiento de efluentes pretratados anaeróbicamente son los siguientes (van HAAMDEL \& LETTIMGA, 1994):
Separación Sólido/Líquido:

Remoción de SST, DBO/DQO

Tratamiento Biológico Aerobio: remoción de DBO/DQO y/o $\mathbf{M M H}_{4}^{+}$

Físico-Químico ó Tratamiento Aerobio/Anóxico: remoción de $\mathbf{M}$ MTK

Físico- Químico ó Anaerobio/Aerobio:

Oxidación Biológica ó Química: remoción de Fósforo remoción de Olor

Físico / Químico u Oxidación Química: remoción de Color

Irradiación Química: remoción de Patógenos

Cuando el reactor UASB está funcionando en condiciones de estabilidad, presenta buena remoción de materia orgánica biodegradable y prácticamente ninguna eficiencia de remoción de M y P. Cuando el postratamiento es utilizado sólo como unidad de pulimiento para materia orgánica carbonácea y sólidos suspendidos, el efecto del tratamiento anaerobio es favorable pues la mayor fracción de estos componentes es removida en el mismo y por lo tanto los requerimientos en el aerobio son mucho menores, lo que representa menores costos.

Cuando se desea remover nutrientes, es importante considerar las relaciones M/DQO y P/DQO mínimas necesarias para obtener un buen desempeño de esos sistemas depuradores. En este caso, el reactor UASB debe ser usado inicialmente para tratar una parte de las aguas residuales afluentes a la planta de tratamiento y el restante ser encaminado directamente al tratamiento biológico complementario con nitrificación y desnitrificación, de modo que se obtenga materia orgánica necesaria para la desnitrificación.

Cuando se utilizan sistemas aerobios intermitentes tipo lodo activado, esta situación se hace menos crítica pues las relaciones M/DQO y $P / D Q O$ van variando a lo largo de un mismo ciclo 
de operación, lo que hace que el sistema anaerobio anaerobio pueda tratar el volumen total de agua reidual afluente y posteriormente pasarla por el sistema aerobio, alcanzándose buena remoción tanto de la materia orgánica carbonácea como de los nutrientes (TORRES, 2000).

Otra ventaja adicional del uso de un reactor UASB combinado con el sistema aerobio es que el primero puede estabilizar el lodo generado en el tratamiento complementario, eliminando la necesidad de unidades adicionales como el espesador y digestor anaerobio de lodos.

\section{costos}

Los reactores UASB presentan un costo de construcción de 10 - 40 U\$/hab, con un valor medio de 20 - 30 U\$/hab sin incluir el costo del terreno. Los costos de operación y mantenimiento oscilan entre $0.50-2.0$ U\$/hab.año, con un valor medio de 1 - 1.5 U\$/hab. año (PROSAB, 1998).

ALEARTS et al (1993) concluyeron que si el costo del terreno es mayor que $U \$ 8 / \mathrm{m}^{2}$, el sistema UASB seguido de cualquier alternativa de postratamiento, resulta hasta $30 \%$ más barato que el uso del proceso completamente aerobio.

La comparación de los costos de inversión y operación de diferentes sistemas de tratamiento mostró que el sistema de lagunas es la solución más barata si el costo del terreno no excede U\$ $12 / \mathrm{m}^{2}$; por encima de ese valor, el proceso anaerobio combinado con el sistema de lagunas es financieramente más atractivo(GTZ, 1997).

DEAM \& HORAM (1995) compararon los costos de inversión y operación de un sistema compuesto de reactor UASB y laguna de maduración con los sistemas de zanjas de oxidación y laguna facultativa seguida de una laguna de maduración. Hasta un costo del terreno de $U \$ 15 / \mathrm{m}^{2}$, el costo total del primer sistema fue $20 \%$ más barato que el sistema de lagunas y $63 \%$ más barato que el sistema de zanjas de oxidación.
SCHELLIMKOUT(1993) comparó varios sistemas para el tratamiento de agua residual doméstica de una población de 50.000 habitantes del centro de Tamiyah en Egipto: UASB, filtro percolador, zanja de oxidación, sistema de lagunas y UASB combinado con filtro percolador o con lagunas. En la evaluación encontró que el costo de inversión del sistema UASB fue el más bajo y los costos de implementación del mismo combinado con filtro percolador o con lagunas como unidades de postratamiento, fueron más bajos que los costos de tales sistemas operando independientemente.

TARE, AHAMMED \& JAWED(1997), compararon el uso del reactor UASB con otros sistemas de tratamiento: lodo activado convencional y de aireación extendida y laguna aireada. En términos de costos de inversión y de operación, el UASB fue el más barato y las lagunas aireadas ocuparon el segundo lugar. En términos de costos anuales de operación y mantenimiento, el UASB fue el más barato de los 4 métodos debido a la exigencia mínima de energía, pues solo se requería para la pos- aireación del efluente del reactor anaerobio.

Los resultados mostrados con relación a los costos de diferentes sistemas de tratamiento comparados con los de los sistemas anaerobios, principalmente de reactores UASB, muestran que también es ventajoso desde el punto de vista económico explorar la potencialidad de los sistemas anaerobios tipo UASB seguidos de alguna alternativa de postratamiento aerobio.

\section{POSTRATAMIENTO DE LOS EFLUENTES DE REACTORES ANAEROBIOS TIPO UASB}

La posibilidad de alternativas de postratamiento de efluentes de reactores anaerobios es bien amplia. Sin embargo, la implementación de algunas combinaciones específicas ha sido más estudiada y aplicada. A continuación se muestran las opciones más comunes: 
UASB-LODO ACTIVADO: van HAAMDEL \& LETTIMGA (1994) evaluaron la conversión de un sistema convencional de lodos activados a un sistema anaerobio-aerobio, utilizando el digestor de lodo del sistema convencional aerobio como la nueva unidad de tratamiento anaerobio del sistema combinado.

Esa conversión mostró una significativa reducción de la materia orgánica y los sólidos suspendidos en el tratamiento anaerobio; de este modo, tanto la demanda de $\mathrm{O}_{2}$ como la masa y producción de lodo en la parte aerobia del sistema combinado, disminuyeron considerablemente. Esa nueva configuración permitió verificar que el volumen de tanques en el sistema combinado puede ser $48 \%$ del volumen de los tanques del sistema aerobio convencional y que el consumo de oxígeno y la producción de lodo se reducen en proporción directa a las remociones adicionales de $\mathrm{DBO}$ en la fase anaerobia.

En términos de consumo de energía para la aireación, éste se reduce entre aproximadamente 50 a $55 \%$ del consumido en el sistema de tratamiento aerobio convencional cuando no se tiene nitrificación y hasta 65 a $70 \%$ cuando se tiene nitrificación (van HAAMDEL, 1998).

UASB-LAGUMA FACULTATIVA: la Corporación Autónoma Regional para la Defensa de la Meseta de Bucaramanga (CDMB), Colombia, luego de varios años de investigación, diseñó, construyó y puso en marcha una planta de tratamiento de aguas residuales domésticas para una población equivalente a 163.000 habitantes, con proyección a 320.000 habitantes; el sistema comprende tratamiento preliminar, 3 reactores UASB (TRH $5.2 \mathrm{~h}$, COV $2 \mathrm{~kg} \mathrm{DQO} / \mathrm{m}^{3} / \mathrm{d}$ ), 2 lagunas facultativas (H $1.5 \mathrm{~m}$, tasa superficial $500 \mathrm{~kg}$. DBO/hab dy TRH 1.25 d).

Dada la alta variación que presenta el flujo de aguas residuales afluente, las lagunas sirven para regular y homogenizar el efluente final removiendo los sólidos suspendidos que periódicamente son arrastrados con el efluente de los reactores anaerobios. En las lagunas ocurre 15 y 25 \% de la remoción global de DBO y SS del sistema respectivamente; el sistema completo alcanza eficiencias de $85 \%$ DBO y $90 \%$ 55, alcanzando los patrones de emisión establecidos para Riofrío que es el cuerpo de agua que recibe el efluente del sistema (RODRÍGUEZ, 1996).

\section{UASB-LODO ACTIVADO IMTERMITEMTE} SALOMÃO (1996) estudió en escala piloto el uso del sistema de lodo activado intermitente como unidad de postratamiento de agua residual doméstica tratada previamente en un reactor UASB de $120 \mathrm{~m}^{3}$. El principal objetivo del estudio fue evaluar las condiciones de operación apropiadas en el RSB para lograr remoción de nutrientes; con un tiempo de aireación de $3 \mathrm{~h}$ y edad de lodo de 10 días, el reactor aerobio mostró buen desempeño con relación a la eficiencia de remoción de DQO, SS y nitrógeno $(60,65$ y $80 \%$ respectivamente) y alguna remoción de organismos patógenos. La remoción biológica de fósforo no ocurrió debido a la ausencia de fases anaerobias durante el tiempo de llenado.

UASB-FILTRO AMAEROBIO: Aunque el filtro anaerobio es una unidad que operando independientemente produce efluentes similares al reactor UASB, ha sido regla general la combinación del tanque séptico seguido de filtro anaerobio. En el estado de Minas Gerais Brasil, se está evaluando la posible configuración del filtro anaerobio como unidad de postratamiento de efluentes de reactores UASB. Los resultados de los experimentos desarrollados demostraron que es posible obtener un efluente final de excelentes características en términos de remoción de materia orgánica carbonácea (mayor de $80 \%$, generalmente entre 85 y 95\%) y sólidos suspendidos (concentración por debajo de 25 mg/l (CHERMICHARO, 1999). 
Cuando el filtro anaerobio es usado como unidad de postratamiento, se recomiendan los siguientes criterios y parámetros de diseño:

Tiempo de retención hidráulica mínima (para Qmáx): $\quad 2$ horas

> Tiempo de retención hidráulica medio (para Qmed):

4 horas

Altura del fondo falso: 0.4 a $0.6 \mathrm{~m}$

Altura del medio de soporte: 1.0 a $1.2 \mathrm{~m}$

Altura del borde libre: $0.30 \mathrm{~m}$

Altura total:

1.5 a $1.8 \mathrm{~m}$

UASB-LAGUMA AIREADA: PASSIG, VILLELA \& FERREIRA(1999) presentaron los resultados del primer año de operación de la planta de tratamiento de Aguas Residuales de Piracicaba PTAR Piramicirin-, la cual tiene capacidad para tratar las Aguas Residuales de una población de 100.000 habitantes, con un caudal proyectado hasta el año 2015, de 50 l/s. La estación está compuesta por reactores UASB modulares, seguidos de una laguna aireada y un decantador secundario con sistema de recirculación de lodo para el UASB.

Los reactores anaerobios manejan eficiencias de remoción de DQO del 65\% y una eficiencia de remoción de DBO en el sistema superior a $80 \%$. Adicionalmente, la producción de lodo del sistema es menor comparada con la producida en un sistema de lodo activado convencional.

UASB-LODO ACTIVADO IMTERMITEMTE: el sistema de tratamiento piloto mostró que el reator UASB ( $\theta 6 \mathrm{~h})$ obtuvo eficiencia media de remoción de $\mathrm{DQO}_{T}$ de $65 \%$ y $\mathrm{DQO}_{\mathrm{F}}$ de $82 \%$, SST $66 \%$ y SSV $62 \%$ además de la ocurrencia de una casi completa amonificación (97\%) y remoción de fósforo de $14 \%$. El reactor aerobio, con tiempos de ciclo de 22, 12, 6 y 4h, presentó buenas eficiencias de nitrificación y de fósforo en función de la condición operacional impuesta, alcanzándose en el sistema combinado completa nitrificación para tiempos de aireación mayores a 10h y una significativa remoción biológica de fósforo (72\%) para tiempos de aireación de apenas $2 \mathrm{~h}$. Mo fue posible hallar una condición operacional que favoreciese la remoción simultánea de nitrógeno y fósforo. Las eficiencias de remoción de $\mathrm{DQO}_{T}, \mathrm{DQO}_{\mathrm{F}}$ y $\mathrm{SS}$ que en media fueron de 92, 94 y $87 \%$ respectivamente, no fueron afectadas significativamente por los cambios en las condiciones operacionales del reactor aerobio (TORRES, 2000).

\section{CONCLUSIONES}

Actualmente ya se tiene una idea generalizada de que tanto los procesos anaerobios como los aerobios pueden ser aplicados al tratamiento de aguas residuales, cada uno de los cuales presentan una serie de aspectos positivos y negativos. Por lo tanto, en cada caso en particular se deben evaluar ambas posibilidades para que se llegue realmente a la solución más apropiada pues la solución escogida deberá corresponder a una eficiencia y costos compatibles en lo posible con las características y circunstancias que prevalecen en el lugar.

Los sistemas anaerobios presentan un gran potencial de aplicación para el tratamiento de aguas residuales domésticas, principalmente en regiones con temperaturas superiores a los $20^{\circ} \mathrm{C}$, con eficiencias de remoción significativas en términos de la materia orgánica carbonácea (DBO, DQO) y de los sólidos suspendidos. Sin embargo, su potencialidad para remover nutrientes y microorganismos patógenos es prácticamente despreciable, lo que hace necesaria la implementación de un sistema complementario para alcanzar los niveles de calidad apropiados para la posterior descarga a los cuerpos de agua receptores o para otros usos.

La obtención de efluente con estas características puede conseguirse con ventajas tanto económicas como operacionales por un sistema compuesto de 
reactor UASB seguido de tratamiento biológico aerobio pues se reduce el consumo de energía necesaria para aireación, volúmenes de reactores y el lodo generado, además de ser de menor cantidad, puede ser estabilizado en el mismo propio reactor UASB, eliminando la necesidad de unidades adicionales como espesadores y digestores de lodo.

Considerando los beneficios generales que ofrece la combinación de los sistemas anaerobios seguidos de los aerobios, se considera que esa nueva concepción tendrá mayor aplicación en el campo del tratamiento de las aguas residuales en general.

\section{REFERENCIAS BIBLIOGRÁFICAS}

DEAM, C; HORAM, M.J. (1995) Aplications of UASB technology in Mauritius. England, 91 p. Research monographs in tropical public health engineering Mo. 7 (PhD) University of Leeds Department of Civil Engineering.

GTZ, sectorial project (1997). Anaerobic trends. IAWQ conference review. WQI, July/august, p. 3133.

van Haandel A.C. \& Lettinga G.(1994). Anaerobic Sewage Treatment. A practical guide for regions with a hot climate. Chichester, England: John Wiley $\&$ Sons Ltd.

van Haandel A.C.; MARAIS, G. (1999). O comportamento do sistema de lodo ativado. Teoria e aplicações para projetos e operação. Epigraf, Brasil.

PASSIG, F.H.; VILLELA, L.C.H.; FERREIRA, O.P. (1999). ETE-Piracimirim- Mova conceição de sistema de tratamento de esgotos sanitários partida, operação e monitoramento de desempenho (I-110). In: $20^{\circ}$ Congresso brasileiro de engenharia sanitaria e ambiental ABES, Rio de Janeiro Brasil, 8p.
PROSAB (1998) Tratamento de esgotos sanitários por processo anaeróbio e disposição controlada no solo. Programa de Pesquisa em Saneamento Básico. ABES RJ, Brasil.

SALOMÃO, Jr.A. (1996). Post-treatment of UASB effluent using SBR system. Delft, The Metherlands, 92p. Thessis (MsC) International Institute for Infrastructural, Hydraulic and Environmental Engineering, IHE.

SCHELLIMKOUT, A. (1993). UASB technology for sewage treatment: experience vith a full scale plant and its applicability in Egypt. Journal Water Science and Technology, v.27, n.9, p.173-180.

TARE, V; AHAMMED, M; JAWED, M. (1997). Biomethanation in domestic and industrial waste treatment an indian scenario. In: $8^{\text {TH }}$ IMTERMATIOMAL COMFEREMCE OM AMAEROBIC DIGESTIOM. V.2. Japan. Proceedings. 255-262.

TORRES, P. (2000). Tratamento de esgoto sanitário em sistema combinado anaeróbioaeróbio (UASB-RSB). São Carlos, 136p. Tese (Doutorado) Escola de Engenharia de São Carlos, Universidade de São Paulo.

VIÑAS, M. (1996). El postratamiento en los sistemas anaerobios. In: IV Seminario-Taller Latinoamericano Tratamiento anaerobio de aguas residuales. Bucaramanga, Colombia, p.471-480. 